\title{
Optics laboratory experiments with laser-heated samples of crude oils and oil-in-water emulsions
}

\section{Germán Da Costa}

Germán Da Costa, "Optics laboratory experiments with laser-heated samples of crude oils and oil-in-water emulsions," Proc. SPIE 9665, Tenth International Topical Meeting on Education and Training in Optics and Photonics, 966527 (3 June 2007); doi: 10.1117/12.2207924

SPIE Event: Tenth International Topical Meeting on Education and Training in Optics and Photonics, 2007, Ottawa, Ontario, Canada 


\section{OPTICS LABORATORY EXPERIMENTS WITH LASER-HEATED SAMPLES OF CRUDE OILS AND OIL-IN-WATER EMULSIONS \\ Germán Da Costa, University Simón Bolivar, Caracas, Venezuela}

The aim of the present paper is to describe incorporation of basic industrial research results into current University study programs in Physics and Optoelectronic Engineering. The Laboratory of Optics and Fluids (LOF) of University Simon Bolivar (USB) leads a research program on applications of Photonics technology in the Petroleum Industry. More precisely, the main research subject at the (LOF) is development of optical procedures allowing determination of conditions of stability of oil-in-water emulsions. In several countries (for example, Canada and Venezuela) there exist important reservoirs of heavy crude oils, whose high viscosity impede their transportation through pipelines. Therefore, emulsions of heavy oils in water were developed in order to allow their commercialization. Though those emulsions are stable in current environmental conditions, high temperature or velocity gradients frequently provoke their coalescence. In typical experiments conducted at the (LOF) temperature gradients are induced in oil-water emulsions and in crude oil samples by irradiation with a CW laser beam. In crude oil samples the strong dependence of the liquid surface tension and refractive index on the local liquid temperature gives rise to long-range deformation of the liquid free surface. The latter cited thus behaves as an interferometrically smooth liquid mirror, which gives rise in turn to phase and intensity variations in the reflected light beam. In emulsion samples the inhomogeneous heating gives rise to thermoconvective flow, which is clearly observed as a moving speckle pattern in the reflected light beam. These are typical phenomena of self-interaction of a laser beam incident upon a material medium. In the present paper we discuss these optical phenomena, first studied in a basic research context, from an educational viewpoint. Simple experimental setups based in these principles are currently used in Physics and Engineering laboratory courses at the USB. The following experiments are discussed in detail:

a) Obtention of a liquid mirror with shape determined at will by the light intensity distribution in a laser beam incident upon a crude oil sample.

b) Study of statistical properties of laser speckle patterns resulting from scattering of laser beams incident upon oil-in-water emulsion samples.

c) Development of optical techniques allowing real time measurement of crude oil refractive index as a function of the liquid temperature.

Tenth International Topical Meeting on Education and Training in Optics and Photonics, edited by Marc Nantel, Proc. of SPIE Vol. 9665, 966527 • (C) 2007 SPIE, OSA, IEEE, ICO doi: $10.1117 / 12.2207924$ 